\title{
On Polynomial Eigenfunctions of a Hypergeometric-Type Operator
}

Gisli Másson and Boris Shapiro

\section{CONTENTS}

1. Introduction

2. Conjectures

3. Proofs

Appendix: Proof of Conjecture 1, by S. Shadrin Acknowledgement

References
Consider the operator

$$
\mathfrak{d}_{Q}(f)=\frac{d^{k}}{d x^{k}}(Q(x) f(x)),
$$

where $\mathrm{Q}(\mathrm{x})$ is some fixed polynomial of degree $\mathrm{k}$. One can easily see that $\mathfrak{d}_{\mathrm{Q}}$ has exactly one polynomial eigenfunction $\mathrm{p}_{\mathrm{n}}(\mathrm{x})$ in each degree $n \geq 0$ and its eigenvalue $\lambda_{n, k}$ equals $(n+k) ! / n$ !. A more intriguing fact is that all zeros of $p_{n}(x)$ lie in the convex hull of the set of zeros to $\mathrm{Q}(\mathrm{x})$. In particular, if $\mathrm{Q}(\mathrm{x})$ has only real zeros then each $p_{n}(x)$ enjoys the same property. We formulate a number of conjectures on different properties of $p_{n}(x)$ based on computer experiments as, for example, the interlacing property, a formula for the asymptotic distribution of zeros etc. These polynomial eigenfunctions might be thought of as a generalization of the classical Gegenbauer polynomials with half-integer superscript, this case arising when our $Q(x)$ is an integer power of $x^{2}-1$.

\section{INTRODUCTION}

A real polynomial in one variable is called hyperbolic if all its zeros (counted with multiplicities) are real. Different properties of hyperbolic polynomials and criteria for hyperbolicity were studied extensively in the beginning of the century; see, for example, [Pólya and Szegö 1976, Chapters 5 and 6]. In the 1960 s and 1970s the interest to hyperbolic polynomials (mostly in the case of several variables) was revitalized due to the fundamental contributions of I. G. Petrovsky and L. Hörmander to the theory of linear partial differential equations with constant coefficients. But some new results were obtained even in the case of one variable; see [Nuij 1968], for example. Later V. Arnol'd and his students wrote a number of papers on hyperbolic polynomials motivated by their application to potential theory [Arnol'd 1982; 1986; Kostov 1989; 1999]. 
A typical context in which hyperbolic polynomials appear is the spectral theory of Sturm-Liouville problems or, more specifically, the theory of orthogonal polynomials; see [Chihara 1978], for example. Namely, given some nonnegative supported on an interval $[a, b]$ weight function $w(x)$ with $\int_{a}^{b} w(x) d x>0$ one gets a family of monic polynomials $\left\{r_{n}(x)\right\}$, $\operatorname{deg} r_{n}(x)=n$, satisfying a condition

$$
\int_{a}^{b} w(x) r_{i}(x) r_{j}(x) d x=\delta_{i, j} .
$$

It is well known that each $r_{i}(x)$ is hyperbolic and all its zeros lie on $[a, b]$. The classical polynomial families such as Hermite, Laguerre, Jacobi polynomials (and their special cases such as Chebyshev, Legendre and Gegenbauer polynomials) arise in this way. Besides hyperbolicity among the main properties of orthogonal polynomials one should mention that

(I) all the zeros of $r_{i}(x)$ are simple (and real);

(II) the zeros of any pair of consecutive orthogonal polynomials $\left(r_{i}(x), r_{i+1}(x)\right)$ are interlacing; that is, $\bar{\alpha}_{1}<\alpha_{1}<\bar{\alpha}_{2}<\alpha_{2}<\cdots<\bar{\alpha}_{i-1}<\alpha_{i-1}<\bar{\alpha}_{i}$, where $\alpha_{j}$ and $\bar{\alpha}_{j}$ are the $j$-th smallest zeros of $r_{i}$ and $r_{j+1}$, respectively;

(III) the density of the asymptotic distribution of zeros of $r_{i}(x)$ when $i \rightarrow \infty$ on the interval $[a, b]$ is independent on the weight function $w(x)$ (supported on $[a, b])$ and is given by

$$
\frac{b-a}{2 \pi \sqrt{(b-x)(x-a)}}
$$

see [Nevai 1979], for example.

In this article we partially prove and mostly conjecture similar results for the polynomial eigenfunctions of the operator

$$
\mathfrak{d}_{Q}(f)=\frac{d^{k}}{d x^{k}}(Q(x) f),
$$

where $Q(x)$ is a polynomial of degree $k$. This operator can be considered as a generalization of the famous hypergeometric operator

$$
\left(x^{2}-1\right) f^{\prime \prime}+(a x+b) f^{\prime}+c .
$$

See also Conjecture 14 in Section 2. Note that most of the abovementioned families of orthogonal polynomials are among its polynomial solutions.
The intriguing detail is that, in general, $\mathfrak{d}_{Q}$ is neither a positive-definite nor selfadjoint operator in which case the above properties are expected.

Convention. Unless mentioned explicitly, $Q$ is always a monic polynomial of degree $k$. For a given $Q$, we denote by $\alpha$ the minimal root and by $\beta$ the maximal root of $Q(x)$, and further, when we use these letters, we assume all the roots of $Q$ are real.

The main results are as follows.

Theorem 1. (a) For any $Q(x)$, the operator $\mathfrak{d}_{Q}$ has one polynomial eigenfunction $p_{n}(x)$ in each degree $n \geq 0$, with eigenvalue $\lambda_{n, k}=(n+k) ! / n !$.

(b) For any $n \geq 0$ all the zeros of $p_{n}(x)$ belong to the convex hull $\mathrm{Conv}_{Q}$ of the set of zeros of $Q(x)$.

Corollary 2. If $Q(x)$ is hyperbolic, each $p_{n}(x)$ is also hyperbolic and its zeros lie between the extreme roots $\alpha$ and $\beta$ of $Q(x)$.

Theorem 3. If $Q(x)$ is hyperbolic and $\alpha<\beta$ (which is to say, $Q(x)$ has distinct roots), the zeros of $p_{n}(x)$ not only belong to $(\alpha, \beta)$ but are all simple.

We now provide explicit determinantal formulas for the coefficients of $p_{n}(x)$. Set

$$
\begin{aligned}
Q(x) & =x^{k}+q_{k-1} x^{k-1} \ldots+q_{1} x+q_{0}, \\
p_{n}(x) & =x^{n}+a_{n, n-1} x^{n-1}+\cdots+a_{n, 1} x+a_{n, 0},
\end{aligned}
$$

where

$$
\begin{aligned}
\frac{d^{k}}{d x^{k}}\left(Q(x) p_{n}(x)\right) & =\lambda_{n, k} p_{n}(x), \\
\lambda_{n, k} & =\frac{(n+k) !}{n !} .
\end{aligned}
$$

Define an upper triangular $(n \times n)$-matrix $M_{n}$ as

$$
\left(\begin{array}{cccccccc}
1-\frac{\lambda_{n, k}}{\lambda_{0, k}} & q_{k-1} & q_{k-2} & \cdots & q_{0} & 0 & \cdots & 0 \\
0 & 1-\frac{\lambda_{n, k}}{\lambda_{1, k}} & q_{k-1} & q_{k-2} & \cdots & q_{0} & \cdots & 0 \\
\vdots & \ddots & \ddots & \ddots & \ddots & \ddots & \vdots \\
& & & & & & q_{0} \\
& & & & & \ddots & \vdots \\
\vdots & & & & & \ddots & q_{k-2} \\
0 & \cdots & & & & & 0 & 1-\frac{\lambda_{n-k}}{\lambda_{n-1, k}}
\end{array}\right)
$$


Theorem 4. For every $n \geq 1$ the coefficients of $p_{n}(x)$ satisfy the linear system

$$
M_{n} A=B
$$

where

$$
A=\left(\begin{array}{c}
a_{n, 0} \\
a_{n, 1} \\
\vdots \\
\vdots \\
\vdots \\
a_{n, n-1}
\end{array}\right), \quad B=-\left(\begin{array}{c}
0 \\
\vdots \\
0 \\
q_{0} \\
q_{1} \\
\vdots \\
q_{k-1}
\end{array}\right)
$$

Proposition 5. If $Q=\left(x^{2}-1\right)^{l}$, the family $\left\{p_{n}(x)\right\}$ coincides up to constant factors with the family of Gegenbauer polynomials with superscript $\gamma=l+\frac{1}{2}$, and with the Jacobi $(l, l)$-polynomials. (The Gegenbauer polynomials $C_{n}^{\gamma}(x)$ may be defined recursively by $C_{0}^{\gamma}(x)=1, C_{1}^{\gamma}(x)=2 \gamma x$, and

$$
C_{n}^{\gamma}(x)=\frac{2(n-1+\gamma)}{n} x C_{n-1}^{\gamma}(x)-\frac{n-2+2 \gamma}{n} C_{n-2}^{\gamma}(x) .
$$

See [Chihara 1978], for example.)

The proofs of these results are given in Section 3 . The next section contains a number of intriguing conjectures.

Remark. After this article was accepted for publication, a number of conjectures presented in Section 2 were proved by $\mathrm{H}$. Rullgård and T. Bergkvist [Bergkvist and Rullgård 2001] and by S. Shadrin; a proof of Conjecture 1 is given here as an appendix. The spectral properties of the operator $\mathfrak{d}_{Q}(f)$ in various functional spaces were recently studied in [Shapiro 2000].

\section{CONJECTURES}

We now formulate a number of intriguing conjectures supported by extensive computer experiments with different choices of $Q$.

Let $\mu_{n}$ denote the discrete probability measure supported on the set of all zeros to $p_{n}(x)$ obtained by placing the mass mult $i / n$ at each of the geometrically distinct roots $x_{i}$ of $p_{n}(x)$, where mult $_{i}$ is the multiplicity of the root. Let $\mu_{Q}=\lim _{n \rightarrow \infty} \mu_{n}$ if it exists. Here convergence is understood as the weak convergence of measures. We call $\mu_{Q}$ the asymptotic measure. Many of our conjectures describe the properties of the support of $\mu_{Q}$ and its density.

\section{Case of Hyperbolic $\mathrm{Q}(\mathrm{x})$}

Extensive computations support a strengthening of Corollary 2:

Conjecture 1. If $Q(x)$ is hyperbolic and has at least two distinct zeros, the zeros of any pair of consecutive polynomial eigenfunctions $p_{i}(x)$ and $p_{i+1}(x)$ are interlacing.

Remark. The proof of this conjecture obtained recently by S. Shadrin has been added with his kind permission to the final version of the paper. See the Appendix below.

Conjecture 2. If $Q(x)$ is hyperbolic of degree $k$, the density of the asymptotic measure $\mu_{Q}$ on the interval $[\alpha, \beta]$ is proportional to $|Q(x)|^{-1 / k}$.

This conjecture is motivated by numerical studies of the distribution of zeros of $p_{n}(x)$. See Figure 1, for example.

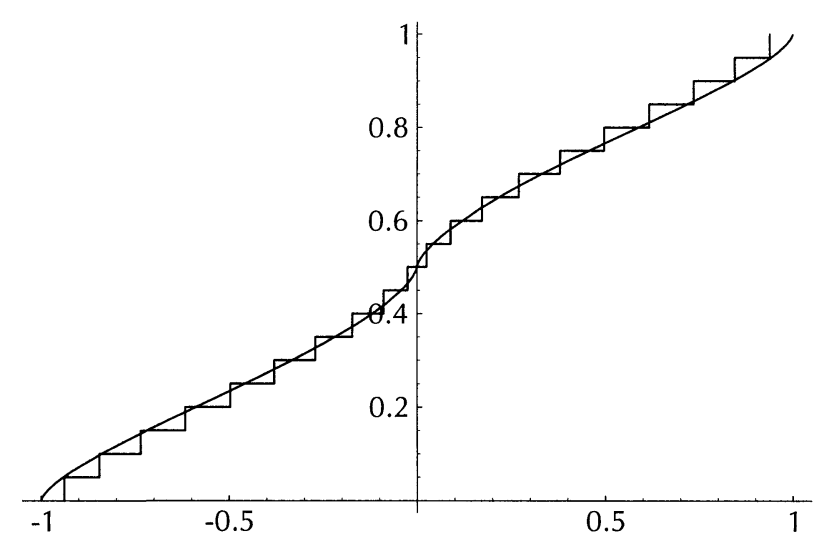

FIGURE 1. Distribution function of the measure $\mu_{20}$ for $Q_{3}(x)=x^{3}-x$, and conjectured asymptotic distribution function $\Gamma\left(\frac{4}{3}\right) /\left(3 \Gamma\left(\frac{2}{3}\right)\right) \int_{-1}^{x}\left|s^{3}-s\right|^{-1 / 3} d s$.

Remark. Suppose Conjecture 2 holds. Then, if $Q(x)$ is not an integer power of a degree 2 polynomial, $\left\{p_{n}(x)\right\}$ is not a system of orthogonal polynomials for some weight function $w(x)$ supported on $[\alpha, \beta]$ or a family obtained by taking derivatives of such, since for such families the asymptotic density of the zeros coincides with

$$
\frac{\beta-\alpha}{2 \pi \sqrt{|(x-\alpha)(x-\beta)|}} .
$$




\section{Case of Real $\mathrm{Q}(\mathrm{x})$}

For fixed $k$ and every $n$, define the discriminantal hypersurface $D_{n} \subset \mathrm{Pol}_{k}^{\mathbb{C}}$ consisting of polynomials $Q$ for which the corresponding eigenpolynomial $p_{n}(x)$ has multiple zeros. Here $\mathrm{Pol}_{k}^{\mathbb{C}}$ is the space of all monic degree $k$ polynomials with complex coefficients; $\mathrm{Pol}_{k}^{\mathbb{R}}$ is defined likewise. Theorem 3 says that if $Q(x)$ is hyperbolic then every $p_{n}(x)$ is hyperbolic and has only simple zeros. Therefore we know that all the discriminants $D_{n}$ do not intersect the domain of strictly hyperbolic polynomials in $\mathrm{Pol}_{k}^{\mathbb{R}}$. An example of these discriminants for the family $Q_{3}(x)=x^{3}+a x+b$ is shown in Figure 2 .

Problem 3. Find equations for $D_{n}$ and study their topological properties.

Conjecture 4. The domain of hyperbolic polynomials is the limit of the intersection of the connected components in the complement to all $D_{n}$ containing this domain.

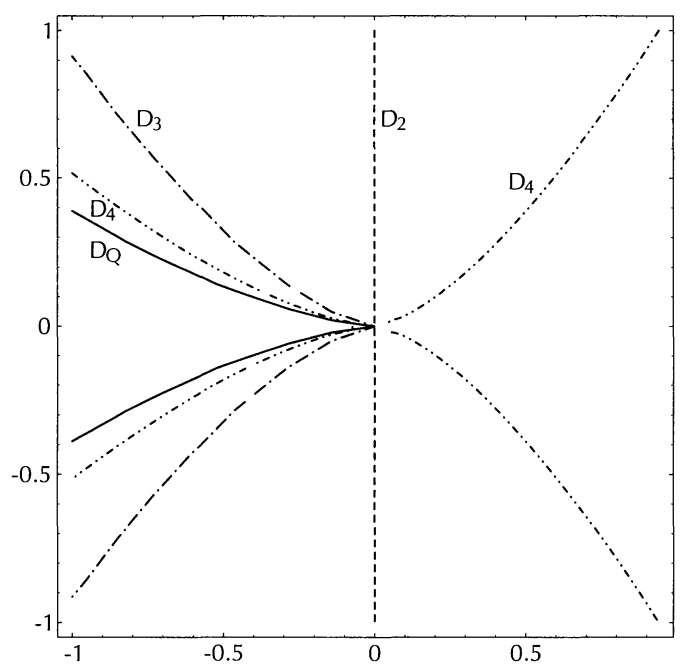

FIGURE 2. Discriminantal curves for $n \leq 4$ in the family $Q_{3}(x)=x^{3}+a x+b$.

Example. The explicit equations of the first three discriminants for the family $Q_{3}(x)=x^{3}+a x+b$ are

$$
\begin{gathered}
D_{2}=\frac{4 a}{9}, \quad D_{3}=\frac{a^{3}}{16}+\frac{27 b^{2}}{361}, \\
D_{4}=\frac{36864 a^{6}}{15353125}+\frac{14336 a^{3} b^{2}}{2042125}-\frac{6912 b^{4}}{923521} .
\end{gathered}
$$

The standard discriminant $D_{Q}$ is given by

$$
D_{Q}=4 a^{3}+27 b^{2} \text {. }
$$

\section{Case of Complex $\mathrm{Q}(\mathrm{x})$}

For a complex polynomial $Q(x)$, we denote by $\mathcal{C}_{Q}$ the support of the asymptotic measure $\mu_{Q}$ and called it the accumulation curve.

Conjecture 5. The accumulation curve $\mathrm{C}_{Q}$ enjoys the following properties:

(a) $\mathcal{C}_{Q}$ is a planar tree imbedded in $\mathrm{Conv}_{Q}$ whose leaves are the roots of $Q(x)$.

(b) For a generic $Q(x)$ the total number of vertices in $\mathcal{C}_{Q}$ equals $2 k-2$, i.e. the number of internal vertices of $\mathrm{C}_{Q}$ equals $k-2$. (About the notion of genericity see Problem 12.)

Select a branch cut $B C$ in $\mathbb{C}$ consisting of rays from all distinct roots of $Q(x)$ to infinity which do not intersect each other or the tree $\mathcal{C}_{Q}$. Select the unique branch of $Q(x)^{-1 / k}$ on the simply connected domain $\Omega_{Q}:=\mathbb{C} \backslash B C$ which asymptotically coincides with $1 / x$ near infinity. Finally choose a point $x_{0}$ in $\Omega_{Q}$ and consider the holomorphic mapping $\Psi_{Q}: \Omega_{Q} \rightarrow$ $\mathbb{C}$ defined by the integral

$$
\Psi_{Q}(x)=\int_{x_{0}}^{x} \frac{d x}{\sqrt[k]{Q(x)}} .
$$

Conjecture 6. $\Psi_{Q}$ maps $\mathcal{C}_{Q}$ onto a planar tree with straight edges. Furthermore, the angles and the densities of the asymptotic measure $\mu(x)$ along edges are uniquely determined by the combinatorics of the tree (see Conjectures 7 and 8).

See Figure 3 for an example.

For a generic $Q$, the image $\Psi_{Q}\left(\mathcal{C}_{Q}\right)$ is independent of the choice of $B C$ as above, and different choices of a base point $x_{0}$ will simply translate the image. (With some straightforward modifications this fact holds for any $Q$.)

Denote by $\Psi \mathcal{C}_{Q}$ the image $\Psi_{Q}\left(\mathcal{C}_{Q}\right)$ of the accumulation curve.

Conjecture 7. The angles at the vertices of $\Psi \mathcal{C}_{Q}$ are defined as follows. Fix a node $v \in \Psi \mathcal{C}_{Q}$. Consider the graph obtained from $\Psi \mathrm{C}_{Q}$ by removing all the edges adjacent to $v$. This graph is a forest whose subtrees are in one-to-one correspondence with the set of these edges. We set the weight \# $(e, v)$ of each such edge e with respect to the vertex $v$ equal to the number of leaves in its corresponding subtree. Then 

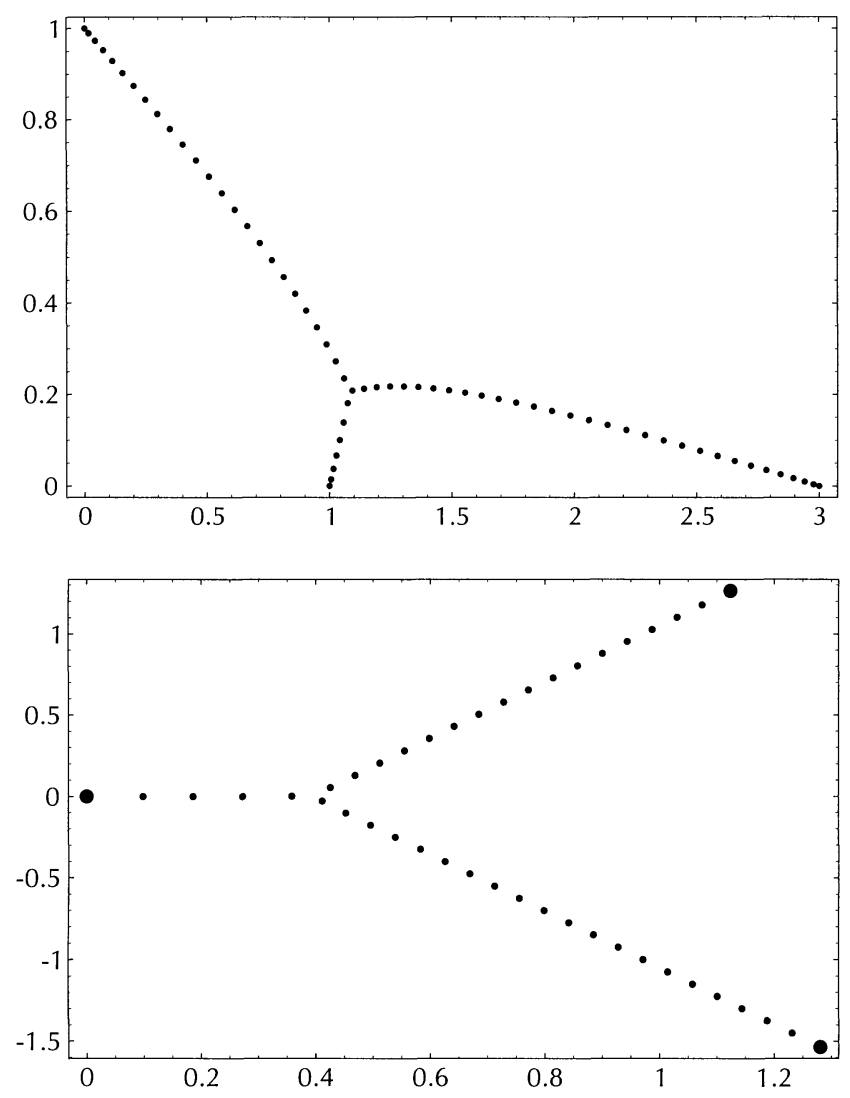

FIGURE 3. Accumulation curve for the polynomial $Q_{3}(x)=(x-1)(x-3)(x-i)$ before and after the mapping $\Psi_{Q}$. (Note that the different horizontal and vertical scales affect the angles between edges.)

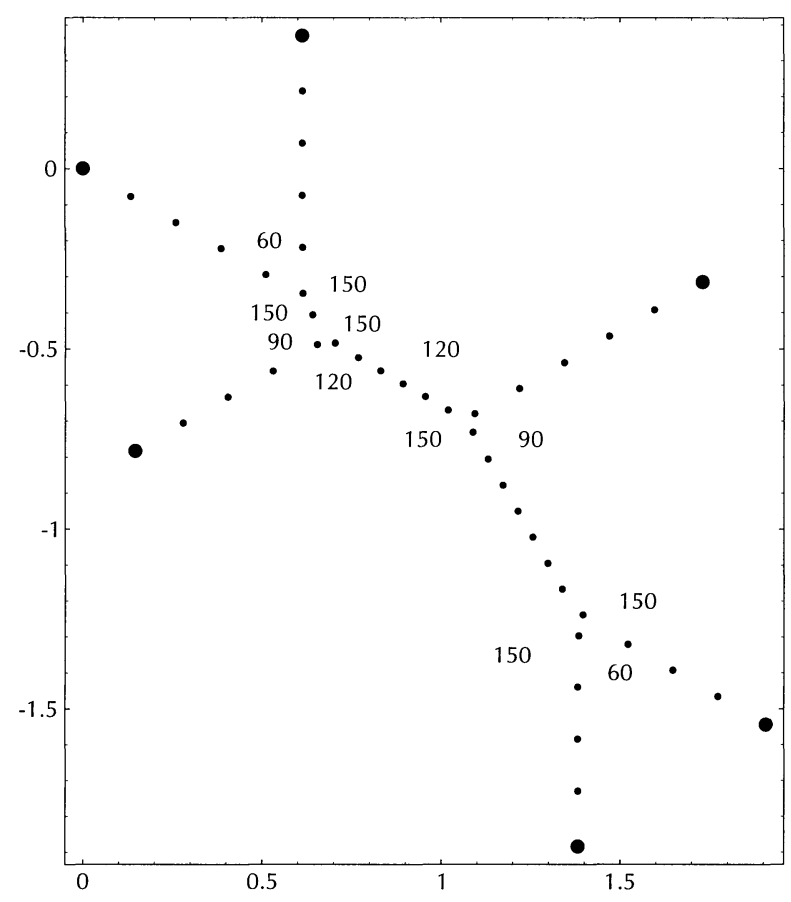

FIGURE 4. How to determine angles. the angle $\varepsilon\left(e_{1}, e_{2}\right)$ between the neighboring edges $e_{1}$ and $e_{2}$ adjacent to $v$ equals

$$
\varepsilon\left(e_{1}, e_{2}\right)=\frac{\pi}{k}\left(\#\left(e_{1}, v\right)+\#\left(e_{2}, v\right)\right) .
$$

See an example in Figure 4.

Let $\Psi \mu_{Q}$ denote the image of the asymptotic measure $\mu_{Q}$ under the transformation $\Psi_{Q}$. Its support obviously coincides with $\Psi \mathcal{C}_{Q}$.

The properties of $\Psi \mu_{Q}$ are completely determined in the following conjecture.

Conjecture 8. The asymptotic distribution $\Psi \mu_{Q}$ of zeros on $\Psi \mathrm{C}_{Q}$ enjoys the following properties.

(a) The density $\nu(e)$ of $\Psi \mu_{Q}$ is constant on every edge e of $\Psi \mathcal{C}_{Q}$, and so equals $\nu(e)=\mu(e) /|e|$, where $\mu(e)$ is the total mass of the edge e and $|e|$ is its length.

(b) For a pair $(v, e)$, where $v$ is some vertex and $e$ is an edge adjacent to $v$ let $\bar{U}(v, e)$ denote the unit vektor parallell to e and pointing towards $v$. Then for any vertex $v$ one has the vector equation

$$
\sum_{e \in E(v)} \nu(e) \bar{U}(v, e)=0
$$

where $E(v)$ is the index set of all edges adjacent to $v$.

For a generic $Q(x)$, these equations give $2(k-2)$ linear equations in $2 k-3$ variables $\nu(e)$, which together with the condition $\sum_{e \in E} \nu(e)|e|=1$ allow us to determine the densities $\nu(e)$ uniquely.

With some straightforward modifications the same recipe works for all $Q$.

Assuming that conjectures 1-8 hold, it is clear that one gets in many respects the same asymptotic behaviour of $p_{n}(x)$ if $Q(x)$ is replaced by its arbitrary integer power $Q^{r}(x)$. Therefore it is reasonable to study the solutions of the equation one gets when taking $r=1 / k$. In that case the "degree" of the polynomial is equal to 1 and the corresponding differential operator is thus of order 1. Furthermore $\lambda_{k, n}=(n+1) \ldots(n+k)$ so for $k=1$ the "correct" eigenvalue for the $n$-th eigenfunction is $(n+1)$. In other words, we want to compare the eigenpolynomials $p_{n}$ of the original operator to the solutions $y_{n}$ of the differential equation

$$
\left(\sqrt[k]{Q y_{n}}\right)^{\prime}=(n+1) y_{n}
$$


Multiplying through by $\sqrt[k]{Q}$ and setting $f_{n}=\sqrt[k]{Q} y_{n}$ transforms this equation into $f_{n}^{\prime}=(n+1) Q^{-1 / k} f_{n}$, or

$$
\operatorname{dlog} f_{n}:=\frac{f_{n}^{\prime}}{f_{n}}=(n+1) Q^{-1 / k} .
$$

Now $f_{n}=Q^{1 / k} y_{n}$ so $\operatorname{dlog} f_{n}=\operatorname{dlog} y_{n}+(1 / k) \operatorname{dlog} Q$ and therefore we get

$$
\frac{1}{n} \mathrm{~d} \log y_{n}=\frac{n+1}{n} Q^{-1 / k}-\frac{1}{k n} \operatorname{dlog} Q .
$$

This implies that $\lim _{n \rightarrow \infty}\left(\operatorname{dlog} y_{n}\right) / n=Q^{-1 / k}$. Since $y_{n}$ corresponds to $p_{n}$ it is therefore natural to expect:

Conjecture 9. In $\mathbb{C} \backslash \mathfrak{C}_{Q}$,

$$
\frac{1}{n} \lim _{n \rightarrow \infty} \frac{p_{n}^{\prime}(x)}{p_{n}(x)}=\frac{1}{\sqrt[k]{Q(x)}}
$$

uniformly in compact neighbourhoods.

Computer experiments suggest that this is indeed true.

By solving the first-order equation above, one gets

$$
f_{n}(x)=e^{(n+1) \Psi_{Q}(x)}
$$

or

$$
y_{n}(x)=\frac{e^{(n+1) \Psi_{Q}(x)}}{\sqrt[k]{Q(x)}}
$$

It is of course not reasonable to expect that $y_{n}(x)$ approaches $p_{n}(x)$ in any sense (note that $y_{n}(x)$ has no roots!). However, the ratio between two consequtive eigenfunctions may be asymptotically the same, that is:

Conjecture 10. In $\mathbb{C} \backslash \mathcal{C}_{Q}$,

$$
\lim _{n \rightarrow \infty} \frac{p_{n+1}(x)}{p_{n}(x)}=e^{\Psi_{Q}(x)}
$$

uniformly in compact neighbourhoods.

An even stronger form of this conjecture is:

Conjecture 11. In $\mathbb{C} \backslash \mathcal{C}_{Q}$,

$$
\lim _{n \rightarrow \infty} \frac{p_{n}(x)}{\left(e^{\Psi_{Q}(x)}\right)^{n}}=\left(\frac{e^{\Psi_{Q}(x)}}{\sqrt[k]{Q}}\right)^{(k+1) / 2}
$$

uniformly in compact neighbourhoods.

Remark. Conjectures 2 and 5-11 have been proved [Bergkvist and Rullgård 2001].

Call a polynomial $Q \in \operatorname{Pol}_{k}^{\mathbb{C}}$ degenerate if its accumulation tree $\mathcal{C}_{Q}$ is not a trivalent graph with $2 k-2$ distinct vertices. The set of all degenerate polynomial form a real hypersurface $\operatorname{Deg}_{k} \subset \mathrm{Pol}_{k}^{\mathbb{C}}$ containing the usual discriminant Disc ${ }_{k}$ consisting of all polynomials with multiple zeros. In the simplest case $k=3$ the discriminant $\operatorname{Deg}_{3}$ consists of all third degree polynomials whose zeros lie on some real affine line in $\mathbb{C}$.

Problem 12. Find the equation for $\operatorname{Deg}_{k}$.

In the first nontrivial case $k=4$ our computer experiments led us to:

Conjecture 13. $\mathrm{Deg}_{4}$ is the analytic continuation of the set of all convex 4-tuples of points on $\mathbb{C}$ such that their convex hull admits an inscribed circle.

The last conjecture deals with a more general class of operators. Take a $(k+1)$-tuple of polynomials $Q_{k}, Q_{k-1}, \ldots, Q_{0}$, where $Q_{k}$ is monic of degree $k$ and every other $Q_{i}$ is of degree at most $i$. Define the differential operator $\mathfrak{d}_{Q_{k}, Q_{k-1}, \ldots, Q_{0}}=\mathfrak{d}_{Q_{k}}+\mathfrak{d}_{Q_{k-1}}+\cdots+$ $\mathfrak{d}_{Q_{0}}$.

Conjecture 14. Conjectures 5-13 hold for $\mathfrak{d}_{Q_{k}, Q_{k-1}, \ldots, Q_{0}}$ as well.

Remarks. 1 . The accumulation curve $\mathcal{C}_{Q}$ introduced above has a strong resemblance with Stokes lines occurring in the theory of differential equations with small parameter and asymptotic expansions, [Coddington and Levinson 1955; Wasow 1985]. The role of a small parameter in our case is played by $1 / \lambda_{n, k}$ when $k$ is fixed and $n \rightarrow \infty$. Using the asymptotic expansion methods the first author was able to prove Conjecture 9 in some neighborhood of infinity.

2. As mentioned to the second author by M. Kontsevich the integral $\Psi_{Q}(x)=\int_{x_{0}}^{x} Q(x)^{-1 / k} d x$ considered on the plane curve $y^{k}=Q(x)$ apparently has properties similar to the properties of Strebel differentials and might serve as their generalization.

\section{PROOFS}

Proof of Theorem 1. Let $\operatorname{Pol}_{\leq m}^{\mathbb{C}}$ be the linear space of all polynomials with complex coefficients whose degree is less or equal $m$. To prove (a), note that the action of $\mathfrak{d}_{Q}$ in the natural basis $x^{m}, x^{m-1}, \ldots, 1$ of $\mathrm{Pol}_{\leq m}^{\mathbb{C}}$ is given by an upper triangular matrix with nonzero diagonal entries $\lambda_{n, k}=(n+k) \ldots(n+1)$ for any $n \leq m$. Thus for each $n \geq 0$ there exists and unique monic polynomial $p_{n}(x)$ which is the 
eigenfunction of $\mathfrak{d}_{Q}$ corresponding to the eigenvalue $\lambda_{n, k}=(n+k) ! / n !$.

In order to prove (b) recall that by the well-known Gauss-Lucas theorem the zeros of $\varphi^{\prime}$ lie in the convex hull $\operatorname{Conv}_{\varphi}$ of zeros to $\varphi$ where $\varphi$ is a polynomial in 1 variable with complex coefficients. Moreover, any zero of $\varphi^{\prime}$ lying on the boundary of $\operatorname{Conv}_{\varphi}$ is a multiple zero of $\varphi$. Let us now assume that $p_{n}(x)$ contains a zero not lying in $\operatorname{Conv}_{Q}$. Then we can always choose such a zero $\kappa$ of $p_{n}(x)$ which lie on the boundary of the convex hull Conv $\mathrm{Cp}_{n}$ of the zeros of the product $Q(x) p_{n}(x)$. Since $p_{n}(x)$ satisfies $(1-1)$ one gets that $\kappa$ should have the same multiplicity both in $p_{n}(x)$ and

$$
\frac{d^{k}}{d x^{k}}\left(Q(x) p_{n}(x)\right)
$$

which is impossible by the assumption that $\kappa$ does not lie in $\operatorname{Conv}_{Q}$.

Proof. Corollary 2 If $Q(x)$ is hyperbolic with the minimal root $\alpha$ and the maximal root $\beta$ then $\operatorname{Conv}_{Q}=$ $[\alpha, \beta]$. Therefore by Theorem 1 all zeros of $p_{n}(x)$ belong to $[\alpha, \beta]$.

To prove Theorem 3 we need a number of additional statements.

Recall that $p_{n}(x)$ denote a monic eigenpolynomial of degree $n$ for the differential operator $\mathfrak{d}_{Q}=$ $\left(d^{k} / d x^{k}\right) Q(x)$, where $Q(x)$ is a monic polynomial of degree $k$. This means that the polynomials $Q$ and $p_{n}$ satisfy the relation

$$
\frac{d^{k}}{d x^{k}}\left(Q p_{n}\right)=\lambda_{k, n} p_{n}
$$

where $\lambda_{n, k}:=(n+k) ! / n !$.

Lemma 3.1. Write $p_{n}=\overline{p_{n}} x^{n_{0}}$ and $Q=\bar{Q} x^{k_{0}}$ where $\overline{p_{n}}$ and $\bar{Q}$ have nonzero constant terms. If $k_{0}<k$, then $k_{0}+n_{0}<k$ and the polynomial $Q p_{n}$ is of the form

$$
\begin{array}{r}
Q p_{n}=x^{k+n}+c_{k+n-1} x^{k+n-1}+\cdots+c_{k+n_{0}} x^{k+n_{0}} \\
+c_{k-1} x^{k-1}+\cdots+c_{k_{0}+n_{0}} x^{k_{0}+n_{0}}
\end{array}
$$

where $c_{k_{0}+n_{0}} \neq 0$.

Proof. If $n_{0}=0$ there is nothing to prove. We may therefore assume throughout the proof that $n_{0}>0$. Write

$$
\begin{aligned}
Q p_{n} & =\bar{Q} \overline{p_{n}} x^{k_{0}+n_{0}} \\
& =c_{k_{0}+n_{0}} x^{k_{0}+n_{0}}+\text { higher order terms. }
\end{aligned}
$$

By construction we have that $c_{k_{0}+n_{0}} \neq 0$.

Assume that $k \leq k_{0}+n_{0}$. Then

$$
\begin{aligned}
\lambda_{n, k} \overline{p_{n}} x^{n_{0}} & =\frac{d^{k}}{d x^{k}}\left(Q p_{n}\right) \\
& =\frac{d^{k}}{d x^{k}}\left(c_{k_{0}+n_{0}} x^{k_{0}+n_{0}}+\text { h.o.t. }\right) \\
& =c_{k_{0}+n_{0}} \frac{\left(k_{0}+n_{0}\right) !}{\left(k_{0}+n_{0}-k\right) !} x^{k_{0}+n_{0}-k}+\text { h.o.t. } \\
& =c_{k_{0}+n_{0}} \frac{\left(k_{0}+n_{0}\right) !}{\left(k_{0}+n_{0}-k\right) !} x^{n_{0}-\left(k-k_{0}\right)}+\text { h.o.t. }
\end{aligned}
$$

which is impossible by the assumption $k>k_{0}$. Thus we must have $k>k_{0}+n_{0}$.

Choose an $i$ with $0 \leq i<n_{0}$ and write $Q p_{n}=$ $c x^{k+i}+$ other terms. Then

$$
\begin{aligned}
\lambda_{n, k} \overline{p_{n}} x^{n_{0}} & =\frac{d^{k}}{d x^{k}} Q(x)\left(Q p_{n}\right) \\
& =\frac{d^{k}}{d x^{k}} Q(x)\left(c x^{k+i}+\text { other terms }\right) \\
& =c \frac{(k+i) !}{i !} x^{i}+\text { other terms. }
\end{aligned}
$$

Since $i<n_{0}$ while the left hand side is a multiple of $x^{n_{0}}$ this implies that $c=0$. This proves the second claim.

Proposition 3.2. If $Q$ has at least two different roots, all the roots of $p_{n}$ have multiplicity at most $k-1$.

Proof. Select a root of $p_{n}$. Changing the independent variable $x \rightarrow x+\gamma$, we may assume that this root is equal to zero. Since $Q$ has at least two different roots, $Q \neq x^{k}$. Thus Lemma 3.1 applies and we have $n_{0} \leq n_{0}+k_{0}<k$.

Remark. We get an even lower bound on the multiplicity in the case when the root of $p_{n}$ is also a root of $Q$.

Proof of Theorem 3. By Proposition 3.2, all the roots of $p_{n}$ are real. Assume that $p_{n}$ has a multiple root. Changing $x \rightarrow x+\gamma$ we may without restriction assume that this root equals zero. Lemma 3.1 thus implies that the polynomial $Q p_{n}$ has at least two consecutive vanishing terms away from the ends. But since all the roots of $Q p_{n}$ are real, this is impossible by Lemma 3.3 below.

The following lemma is almost certainly well known, but we prove it here for the convenience of the reader. 
Lemma 3.3. Let $s=a_{n+m} x^{n+m}+\cdots+a_{m} x^{m}$ be $a$ real polynomial with $a_{n+m} \neq 0 \neq a_{m}$. If $a_{m+i}=$ $a_{m+i+1}=0$ for some $i=1, \ldots, n-2$ then $s$ is not hyperbolic.

Proof. Without loss of generality we may assume that $m=0$. First we treat the special case $s=$ $a_{n} x^{n}+a_{n-r} x^{n-r}+\cdots+a_{0}$ where $r>2$ and $a_{n-r} \neq 0$. Then $s^{(n-r)}$ is not hyperbolic and consequently $s$ is not hyperbolic (since the derivative of a hyperbolic polynomial is hyperbolic).

The special case $s=a_{n} x^{n}+\cdots+a_{r} x^{r}+a_{0}$ where $r>2$ and $a_{r} \neq 0$ can be reduced to the first case by looking at the polynomial $x^{n} s(1 / x)$.

Finally the general case may be reduced to the second case by a suitable number of derivations.

Proof of Theorem 4. The Laplace transform of a polynomial $p(x)$ is

$$
L[p](t)=\int_{0}^{+\infty} e^{-t x} p(x) d x, \quad t>0 .
$$

If a polynomial $p_{n}$ satisfies (1-1), then

$$
L\left[\frac{d^{k}}{d x^{k}}\left(Q p_{n}\right)\right]=L\left[\lambda_{n} p_{n}\right]
$$

Standard properties of Laplace transformation imply that

$$
\begin{aligned}
L\left[\lambda_{n, k} p_{n}\right] & =\lambda_{n, k} L\left[p_{n}\right], \\
L\left[\frac{d^{k}}{d x^{k}} Q p_{n}\right] & =t^{k} L\left[Q p_{n}\right]-\left.\sum_{i=0}^{k-1} t^{i} \frac{d^{k-i-1}}{d x^{k-i-1}}\right|_{x=0}\left(Q p_{n}\right), \\
L\left[Q p_{n}\right] & =L\left[\sum_{i=0}^{k} q_{i} x^{i} p_{n}\right]=\sum_{i=0}^{k} q_{i}(-1)^{i} \frac{d^{i}}{d t^{i}} L\left[p_{n}\right] \\
& =Q\left(-\frac{d}{d t}\right)\left[L\left[p_{n}\right]\right], \\
L\left[p_{n}\right] & =L\left[\sum_{i=0}^{n} a_{n, i} x^{i}\right]=\sum_{i=0}^{n} a_{n, i} \frac{i !}{t^{i+1}}, \\
Q\left(-\frac{d}{d t}\right)\left[L\left[p_{n}\right]\right] & =\sum_{i=0}^{n} a_{n, i} i ! Q\left(-\frac{d}{d t}\right)\left[\frac{1}{t^{i+1}}\right] \\
& =\sum_{i=0}^{n} a_{n, i} \sum_{j=0}^{k} q_{j} \frac{(i+j) !}{t^{i+j+1}}, \\
L\left[\frac{d^{k}}{d x^{k}}\left(Q p_{n}\right)\right] & =t^{k} \sum_{0 \leq i \leq n, 0 \leq j \leq k} a_{n, i} q_{j} \frac{(i+j) !}{t^{i+j+1}} .
\end{aligned}
$$

Hence, the coefficients of the polynomial $p_{n}$ satisfy the identity

$\sum_{m=0}^{n} \frac{(m+k) !}{t^{m+1}} \sum_{\substack{m \leq i \leq n \\ i \leq m+k}} a_{n, i} q_{m+k-i}=\lambda_{n, k} \sum_{m=0}^{n} a_{n, m} \frac{m !}{t^{m+1}}$.

It implies the equalities

$$
\sum_{\substack{m \leq i \leq n \\ i \leq m+k}} a_{n, i} q_{m+k-i}=\frac{\lambda_{n, k}}{\lambda_{m, k}} a_{n, m}, \quad m=0, \ldots, n .
$$

The equality for $m=n$ is trivial since $a_{n, n}=q_{k}=1$. The other equalities define the linear system (1-2). Since $\operatorname{det} M_{n}=\prod_{j=0}^{n-1}\left(1-\lambda_{n, k} / \lambda_{j, k}\right) \neq 0$, this system has a unique solution.

Applying Cramer's formulas to the linear system above we get:

Corollary 3.4. The coefficients of $p_{n}(x)$ equal

$$
a_{n, i}=-D_{n, i} \prod_{j=i}^{n-1}\left(1-\frac{\lambda_{n, k}}{\lambda_{j, k}}\right)^{-1}
$$

where $i=0, \ldots, n-1$ and where $D_{n, i}$ is the determinant of the matrix obtained from the upper triangular $(n-i) \times(n-i)$-matrix

$M_{n, i}=$

$$
\left(\begin{array}{cccccccc}
1-\frac{\lambda_{n, k}}{\lambda_{i, k}} & q_{k-1} & q_{k-2} & \cdots & q_{0} & 0 & \cdots & 0 \\
0 & 1-\frac{\lambda_{n, k}}{\lambda_{i+1, k}} & q_{k-1} & q_{k-2} & \cdots & q_{0} & \cdots & 0 \\
\vdots & \ddots & \ddots & \ddots & \ddots & \ddots & \vdots \\
& & & & & & q_{0} \\
& & & & & \ddots & \vdots \\
\vdots & & & & & \ddots & q_{k-2} \\
0 & \cdots & & & & & 0 & 1-\frac{\lambda_{n, k}}{\lambda_{n-1, k}}
\end{array}\right)
$$

by replacing the first column with the numbers

$$
\left(0, \ldots, 0, q_{0}, \ldots, q_{k-2}, q_{k-1}\right) .
$$

Corollary 3.5. If the sum of roots of a polynomial $Q(x)$ vanishes, the same is true for every $p_{n}(x)$ with $n>0$.

Proof. According to Corollary 3.3,

$$
a_{n, n-1}=-\frac{q_{k-1}}{1-\frac{\lambda_{n, k}}{\lambda_{n-1, k}}} \text {. }
$$


Hence, $a_{n, n-1}=0$ if $q_{k-1}=0$.

Corollary 3.6. If $Q(x)$ is even, the polynomials $p_{n}(x)$ with even $n$ are even. Likewise with "even" replaced by "odd".

Proof. According to Corollary 3.3, we have to prove that $D_{n, i}=0$ for all $i=n-1 \bmod 2$ if $q_{j}=0$ for all $j=k-1 \bmod 2$. But for such a polynomial $Q(x)$ and odd $n-i$, the determinant $D_{n, i}$ is equal to

$$
\prod_{\substack{i<j<n-1 \\ j \equiv i+1 \bmod 2}}\left(1-\frac{\lambda_{n, k}}{\lambda_{j, k}}\right) \operatorname{det} \tilde{M}_{n, i},
$$

where the matrix $\widetilde{M}_{n, i}$ is obtained from $M_{n, i}$ by deletion of all even columns and rows. It remains to notice that the first column of the matrix $\widetilde{M}_{n, i}$ vanishes.

Example. It is easy to calculate the coefficients of the polynomials $p_{n}(x)$ in the case when $Q(x)$ has only two terms. Indeed, let $Q(x)=x^{k}-\alpha x^{k-m}$ where $1 \leq m \leq k$. Then the system (1-2) can be easily solved by Gauss elimination:

$$
\begin{gathered}
a_{n, i}=0 \quad \text { if } i \neq n \bmod m, \\
a_{n, n-m l}=-\alpha^{l} \prod_{j=1}^{l}\left(1-\frac{\lambda_{n, k}}{\lambda_{n-m j, k}}\right)^{-1}
\end{gathered}
$$

for all natural $l$ such that $m l<n$.

Proof of Proposition 5. We start with the following statement.

Lemma 3.7. Suppose $Q=\left(x^{2}-1\right)^{l}$, for $l$ a natural number. The eigenpolynomials $p_{n}(x)$ and $p_{m}(x)$, for $n \neq m$, satisfy the orthogonality condition

$$
\int_{-1}^{1}\left(x^{2}-1\right)^{l} p_{n}(x) p_{m}(x) d x=0 .
$$

Proof. Take the polynomial family $\left\{q_{n}\right\}$, where $q_{n}=$ $\left(x^{2}-1\right)^{2 l} p_{n}$. One checks directly that $p_{n}=q_{n}^{(2 l)} / \lambda_{n, 2 l}$ and that $q_{n}$ satisfies $\lambda_{n, 2 l} q_{n}(x)=\left(x^{2}-1\right)^{l} q_{n}^{(2 l)}$. Take

$$
I_{n, m}=\int_{-1}^{1}\left(x^{2}-1\right)^{l} p_{n}(x) p_{m}(x) d x .
$$

In terms of $q_{i}$ 's the integral $I_{n, m}$ can be rewritten as

$$
I_{n, m}=\lambda_{n, 2 l} \int_{-1}^{1} q_{n}^{(2 l)}(x) q_{m}(x) d x .
$$

Both $q_{n}(x)$ and $q_{m}(x)$ have zeros of multiplicity at least $l$ at \pm 1 , so their derivatives of order up to $l-1$ vanish at these points. Integrating by parts $2 l$ times the right-hand side of the integral and using the previous remark we arrive at

$$
\begin{aligned}
I_{n, m} & =\lambda_{n, 2 l} \int_{-1}^{1} q_{n}^{(2 l)}(x) q_{m}(x) d x \\
& =\lambda_{n, 2 l} \int_{-1}^{1} q_{n}(x) q_{m}^{(2 l)}(x) d x=\frac{\lambda_{n, 2 l}}{\lambda_{m, 2 l}} I_{n, m} .
\end{aligned}
$$

Since $0 \neq \lambda_{m, 2 l} \neq \lambda_{n, 2 l} \neq 0$ for $m \neq n$, one has $I_{n, m}=0$. Therefore the family $\left\{p_{n}(x)\right\}$ is orthogonal on the interval $[-1,1]$ with respect to the nonnegative weight function $\left(x^{2}-1\right)^{l}$.

Proposition 5 follows since a system of polynomials orthogonal on $[-1,1]$ with the weight function $Q=$ $\left(x^{2}-1\right)^{l}$ coincides (up to constant factors) with the Gegenbauer polynomials $C_{n}^{\gamma}(x)$ with $\gamma=l+\frac{1}{2}$.

\section{APPENDIX: PROOF OF CONJECTURE 1 BY S. SHADRIN}

The interlacing property of the $p_{n}$ 's in the case when $Q$ has only real zeros was proved by S. Shadrin. The proof consists of three lemmas, of which Lemmas A.1 and A.3 are apparently well known.

Lemma A.8. If $R_{n}$ and $R_{n+1}$ are strictly hyperbolic polynomials of degrees $n$ and $n+1$, respectively, $R_{n}+$ $\varepsilon R_{n+1}$ is hyperbolic for any sufficiently small $\varepsilon$.

Proof. For any sufficiently small $\varepsilon$ the $n$ real zeros of $R_{n}+\varepsilon R_{n+1}$ are located in some small neighborhoods of the $n$ simple real zeros of $R_{n}$ and the $(n+1)$-th real zero has a very big absolute value.

Lemma A.9. If $Q$ has only real zeros, any linear combination $\alpha p_{n}+\beta p_{n+1}$ with real coefficients of the polynomial egenfunctions of $\mathfrak{d}_{Q}$ is a hyperbolic polynomial.

Proof. Applying to $\alpha p_{n}+\beta p_{n+1}$ some high power $\mathfrak{d}_{Q}^{-N}$ of the inverse operator one gets

$$
\begin{aligned}
\mathfrak{d}_{Q}^{-N}\left(\alpha p_{n}+\beta p_{n+1}\right) & =\frac{\alpha}{\lambda_{n, k}^{N}} p_{n}+\frac{\beta}{\lambda_{n+1, k}^{N}} p_{n+1} \\
& =\frac{\alpha}{\lambda_{n, k}^{N}}\left(p_{n}+\varepsilon p_{n+1}\right),
\end{aligned}
$$

where $\varepsilon$ is arbitrarily small for the appropriate choice of $N$ (since $0<\lambda_{n, k}<\lambda_{n+1, k}$ ). Thus, by Lemma A.1, the polynomial $\mathfrak{d}_{Q}^{-N}\left(\alpha p_{n}+\beta p_{n+1}\right)$ is hyperbolic for sufficiently big $N$. Assume that $\alpha p_{n}+\beta p_{n+1}$ is nonhyperbolic and take the largest $N_{0}$ for which 
$R_{N_{0}}=\mathfrak{d}_{Q}^{-N_{0}}\left(\alpha p_{n}+\beta p_{n+1}\right)$ is still nonhyperbolic. Then, obviously, $R_{N_{0}}=\mathfrak{d}_{Q}\left(R_{N_{0}+1}\right)$ where $R_{N_{0}+1}=$ $\mathfrak{d}_{Q}^{-N_{0}+1}\left(\alpha p_{n}+\beta p_{n+1}\right)$. Note that $R_{N_{0}+1}$ is hyperbolic and that if $Q$ has only real zeros then the application of $\mathfrak{d}_{Q}$ to any hyperbolic polynomial results in a hyperbolic polynomial. Contradiction.

Lemma A.10. If $R_{n}$ and $R_{n+1}$ are any real polynomials of degrees $n$ and $n+1$, respectively, saying that every linear combination $\alpha R_{n}+\beta R_{n+1}$ with real coefficients is hyperbolic is equivalent to saying that

(i) both $R_{n}$ and $R_{n+1}$ are hyperbolic, and

(ii) their roots are interlacing.

Proof. Condition (i) is obviously necessary since otherwise one can choose $\alpha=0$ or $\beta=0$ to get a nonhyperbolic polynomial. Assume that both $R_{n}$ and $R_{n+1}$ are hyperbolic and that $x_{i}<x_{i+1}$ are two consecutive zeros of, say, $R_{n}$ not separated by a zero of $R_{n+1}$. Then considering $R_{n}+t R_{n+1}$ where $|t|$ is increasing and the sign of $t$ is chosen so that $t R_{n+1}$ and $R_{n}$ have different signs on $\left(x_{i}, x_{i+1}\right)$, we get that for sufficiently big $|t|$ the polynomial $R_{n}+t R_{n+1}$ looses its hyperbolicity.

On the other hand, (i) and (ii) are sufficient. (We consider only the case of simple zeros of $R_{n+1}$, which implies the general case as well.) Indeed, any linear combination $\alpha R_{n}+\beta R_{n+1}$ with $\alpha \neq 0$ changes its sign at any two consecutive zeros of $R_{n+1}$. Therefore $\alpha R_{n}+\beta R_{n+1}$ has at least $n$ real zeros, and thus all its $n+1$ zeros are real.

\section{ACKNOWLEDGEMENTS}

The authors are grateful to T. Ekedahl, H. Rullgård, H. Shapiro, S. Shadrin and T. Bergkvist for a number of stimulaing discussions of the project.

B. Shapiro acknowledges the hospitality and financial support of the Max-Planck Institute (Bonn) during August and September 2000, and of IHES (Paris) during January 2001, when this article was prepared.

\section{REFERENCES}

[Arnol'd 1982] V. I. Arnol'd, "The Newton potential of hyperbolic layers", Trudy Tbiliss. Univ. 232/233 (1982), 23-29. In Russian.

[Arnol'd 1986] V. I. Arnol'd, "Hyperbolic polynomials and Vandermonde mappings", Funktsional. Anal. $i$ Prilozhen. 20:2 (1986), 52-53. In Russian; translated in Funct. Anal. Appl 20:2 (1986), 125-128.

[Bergkvist and Rullgård 2001] T. Bergkvist and H. Rullgård, "On polynomial eigenfunctions for a class of differential operators", preprint 13, Univ. of Stockholm, 2001. See http://matematik.su.se/reports/2001/ 13. Submitted to Math. Res. Letters.

[Chihara 1978] T. S. Chihara, An introduction to orthogonal polynomials, Math. and its Appl. 13, Gordon and Breach, New York, 1978.

[Coddington and Levinson 1955] E. A. Coddington and N. Levinson, Theory of ordinary differential equations, McGraw-Hill, New York, 1955.

[Kostov 1989] V. P. Kostov, "On the geometric properties of Vandermonde's mapping and on the problem of moments", Proc. Roy. Soc. Edinburgh Sect. A 112:3-4 (1989), 203-211.

[Kostov 1999] V. P. Kostov, "On the hyperbolicity domain of the polynomial $x^{n}+a_{1} x^{n-1}+\cdots+a_{n}$ ", Serdica Math. J. 25:1 (1999), 47-70.

[Nevai 1979] P. G. Nevai, Orthogonal polynomials, Mem. Amer. Math. Soc. 213, Amer. Math. Soc., Providence, RI, 1979.

[Nuij 1968] W. Nuij, "A note on hyperbolic polynomials", Math. Scand. 23 (1968), 69-72.

[Pólya and Szegő 1976] G. Pólya and G. Szegő, Problems and theorems in analysis, II: Theory of functions, zeros, polynomials, determinants, number theory, geometry, Grundlehren der Math. Wissenschaften 216, Springer, New York, 1976.

[Shapiro 2000] H. Shapiro, "Spectral aspects of a class of differential operators", preprint, 2000. Submitted to the Proceedings of Sonya Kovalevsky Conference.

[Wasow 1985] W. Wasow, Linear turning point theory, Appl. Math. Sci. 54, Springer, New York, 1985.

Gisli Másson, deCODE Genetics Inc, Lynghals 1, IS-110, Reykjavik, Iceland (gisli.masson@decode.is)

Boris Shapiro, Department of Mathematics, University of Stockholm, S-10691, Sweden (shapiro@matematik.su.se)

Received February 7, 2001; accepted in revised form June 1, 2001 